\title{
Pseudo-metric space and fixed point theorem
}

\author{
Samih Lazaiz, Karim Chaira, Mohamed Aamri and El Miloudi Marhrani* (D)
}

\author{
"Correspondence: \\ marhrani@gmail.com \\ Laboratory of Algebra Analysis and \\ Applications ( $L 3 A)$, Department of \\ Mathematics and Computer \\ Science, Faculty of Sciences Ben \\ M'Sik, Hassan II University of \\ Casablanca, Sidi Othman, BP 7955, \\ Casablanca, Morocco
}

\begin{abstract}
The aim of this paper is to give a generalized version of Caristi fixed point theorems in pseudo-metric spaces. Our results generalize and improve many of well-known theorems. As an application of our results, we give a new existence theorem to the generalized nonlinear complementarity problem and a solution of differential inclusion in the distributions setting.
\end{abstract}

Keywords: Caristi-type fixed point; Brezis-Browder principle; nonlinear complementarity problem; supernormal cone; differential inclusion

\section{Introduction}

It is well known that the Ekeland variational principle [1] and Caristi-Kirk fixed point theorem are both equivalent. Many authors [2-7] have established a generalized version of these two results in different settings, that is, in vector-valued generalized metric space with respect to a convex cone $\mathbb{K}$ in a Banach space. Recall that a subset $\mathbb{K} \subset \mathbb{Y}$ is called a convex cone on a topological vector space $\mathbb{Y}$ if:

1. $\mathbb{K}+\mathbb{K} \subset \mathbb{K}$

2. for every $\lambda>0, \lambda \mathbb{K} \subset \mathbb{K}$;

3. $\mathbb{K} \cap(-\mathbb{K})=\{\theta\}$, where $\theta$ denotes the zero of $\mathbb{Y}$.

A convex cone $\mathbb{K} \subset \mathbb{Y}$ generates a partial ordering on $\mathbb{Y}$ (i.e. a reflexive, antisymmetric, and transitive relation) by

$$
x \preceq y \quad \Longleftrightarrow \quad y-x \in \mathbb{K} \text {. }
$$

Thereby, since its appearance, the Brezis-Browder ordering principle [8] seems to be a strong tool to prove fixed point or minimal point theorems in an ordered set. Zermelo's theorem [9] shows that there is an equivalency between the existence of a fixed point of such a map and the monotonicity of the map. By the way, Hamel [10] studied existence theorems, namely minimal point, Caristi fixed point, and Ekeland variational principle in the topological product space $\mathbb{X} \times \mathbb{Y}$ where $\mathbb{X}$ is a separated uniform space, and $\mathbb{Y}$ is a topological vector space.

Fang [11] introduced the concept of 'F-type topological spaces' generating the topology by families of quasi-metrics and gave a generalization of Ekeland's variational principle.

Furthermore, Isac [12] proved an interesting Caristi-type theorem in the framework of locally convex space, which led him to derive an existence result of a nonlinear equation.

(c) The Author(s) 2017. This article is distributed under the terms of the Creative Commons Attribution 4.0 International License (http://creativecommons.org/licenses/by/4.0/), which permits unrestricted use, distribution, and reproduction in any medium, provided you give appropriate credit to the original author(s) and the source, provide a link to the Creative Commons license, and indicate if changes were made. 
Hence, the aim of this paper is to generalize some of the well-known fixed point theorems $[11,13-15]$ for a pseudo-metric space $\mathbb{X}$. This paper is divided into three sections after showing some basic results in preliminaries. Using in Section 3 the Brezis-Browder principle, we give generalized Caristi's fixed point theorems for set-valued maps and derive some corollaries. Section 4 is devoted to an Ekeland-type variational principle in more applied general setting, namely pseudo-metric spaces, and also discuss the relationships of our main results. Finally, following investigations by Isac, Section 5 is devoted to applications.

\section{Preliminaries}

Over this section, $\mathbb{Y}$ is a locally convex space, and $\mathbb{K}$ is a convex cone in $\mathbb{Y}$. A set $\Lambda$ is said to be a directed set if ' $\prec$ ' is a preorder and every pair of elements of $\Lambda$ has an upper bound.

Definition 2.1 Let $\mathbb{X}$ be a nonempty set, and $(\Lambda, \prec)$ a directed set. A family of cone pseudo-metrics on $\mathbb{X}$ is a system $\left\{d_{\alpha}\right\}_{\alpha \in \Lambda}$ of mappings $d_{\alpha}: \mathbb{X} \times \mathbb{X} \rightarrow \mathbb{K}$ satisfying the following conditions for each $\alpha \in \Lambda$ and $x, y, z \in \mathbb{X}$ :

(A1) $\theta \preceq d_{\alpha}(x, y)$, and $d_{\alpha}(x, x)=\theta$;

(A2) $d_{\alpha}(x, y)=d_{\alpha}(y, x)$;

(A3) $d_{\alpha}(x, z) \preceq d_{\alpha}(x, y)+d_{\alpha}(y, z)$;

(A4) If $\alpha \prec \beta$ then $d_{\alpha}(x, y) \preceq d_{\beta}(x, y)$.

Then the pair $\left(\mathbb{X},\left\{d_{\alpha}\right\}_{\alpha \in \Lambda}\right)$ is called a cone pseudo-metric space. Additionally, if

(A5) for all $\alpha \in \Lambda$ and $x, y \in \mathbb{X}, d_{\alpha}(x, y)=\theta$ implies $x=y$,

then the family of cone pseudo-metrics is said to be separating.

The concept of a cone pseudo-metric space was already defined by Włodarczyk et al. [16], who called it a Hausdorff cone pseudo-metric space. In this paper, we use a locally convex space as a target set for a cone pseudo-metric, which is more general that a normed space. If $(\mathbb{Y}, \tau)$ is a locally convex space, then it is known that the topology $\tau$ can be generated by a family of seminorms $\left\{p_{i}\right\}_{i \in I}$ [17]. A subset $B$ of $\left\{p_{i}\right\}_{i \in I}$ is called a basis for $\left\{p_{i}\right\}_{i \in I}$ if for every $i \in I$, there exist $q \in B$ and $\lambda>0$ such that $p_{i} \leq \lambda q$.

We say that a family of seminorms $\left\{p_{i}\right\}_{i \in I}$ is separating if $\operatorname{ker}\left\{p_{i}\right\}_{i \in I}=\{\theta\}$ or has a Hausdorff basis $B$ if $\operatorname{ker} B=\{\theta\}$, where

$$
\operatorname{ker} B=\{x \in \mathbb{Y}: p(x)=0, \forall p \in B\} .
$$

The most useful class of cones in topological vector space is the class of normal cones. For more details, we refer the reader to [18].

Definition 2.2 ([13]) If $\left(\mathbb{Y},\left\{p_{i}\right\}_{i \in I}\right)$ is a locally convex space, then a convex cone $\mathbb{K} \subset \mathbb{Y}$ is said to be normal if there exists a basis $B$ of $\left\{p_{i}\right\}_{i \in I}$ such that, for each $p \in B$ and all $x, y \in \mathbb{K}$,

$$
\theta \preceq x \preceq y \quad \Longrightarrow \quad p(x) \leq p(y)
$$

Throughout this paper, we assume that the topology defined on $\mathbb{Y}$ is generated by the basis $B$ [13], and we simply write $B=\left\{p_{i}\right\}_{i \in I}$. 
Proposition 2.3 Let $\left(\mathbb{X},\left\{d_{\alpha}\right\}_{\alpha \in \Lambda}\right)$ be a cone pseudo-metric space over a normal cone $\mathbb{K}$.

Then the mappings $\delta_{\alpha i}: \mathbb{X} \times \mathbb{X} \rightarrow\left[0, \infty\left[\right.\right.$ defined for each $(\alpha, i) \in \Lambda \times I$ by $\delta_{\alpha i}=p_{i} \circ d_{\alpha}$ is a family of pseudo-metrics on $\mathbb{X}$.

Proof By (A1) and (A2) we have immediately $\delta_{\alpha i}(x, x)=0$ and $\delta_{\alpha i}(x, y)=\delta_{\alpha i}(y, x)$ for every $x, y \in \mathbb{X}$.

Since for each $\alpha \in \Lambda$ and all $x, y, z \in \mathbb{X}$, we have $d_{\alpha}(x, y) \in \mathbb{K}$ and

$$
\theta \preceq d_{\alpha}(x, z) \preceq d_{\alpha}(x, y)+d_{\alpha}(y, x)
$$

and since $\mathbb{K}$ is a normal cone, we get, for each $i \in I$,

$$
p_{i}\left(d_{\alpha}(x, z)\right) \leq p_{i}\left(d_{\alpha}(x, y)+d_{\alpha}(y, x)\right) \leq p_{i}\left(d_{\alpha}(x, y)\right)+p_{i}\left(d_{\alpha}(y, x)\right)
$$

Then $\delta_{\alpha i}$ satisfies the triangle inequality. If we assume that $\left\{d_{\alpha}\right\}_{\alpha \in \Lambda}$ is a separating family, so is $\left\{\delta_{\alpha i}\right\}_{(\alpha, i) \in \Lambda \times I}$.

If the convex cone $\mathbb{K}$ is solid (int $\mathbb{K} \neq \emptyset$ ) and not normal and if $\mathbb{Y}$ is a locally convex space, then the Gerstewitz functional [19] $\xi_{e}: \mathbb{Y} \rightarrow \mathbb{R}$, where $e \in \operatorname{int} \mathbb{K}$, is defined as

$$
\xi_{e}(x)=\inf \{\lambda \in \mathbb{R}: x \in \lambda e-\mathbb{K}\}
$$

for each $x \in \mathbb{Y}$.

We have the following result.

Lemma 2.4 For all $\lambda \in \mathbb{R}$ and $x \in \mathbb{Y}$, we have the following statements:

(i) $\xi_{e}(x) \leq \lambda \Longleftrightarrow x \in \lambda e-\mathbb{K}$;

(ii) $\xi_{e}(x)>\lambda \Longleftrightarrow x \notin \lambda e-\mathbb{K}$;

(iii) $\xi_{e}(x) \geq \lambda \Longleftrightarrow x \notin \lambda e-\operatorname{int} \mathbb{K}$;

(iv) $\xi_{e}(x)<\lambda \Longleftrightarrow x \in \lambda e-\operatorname{int} \mathbb{K}$;

(v) $\xi_{e}(\cdot)$ is positively homogeneous and continuous on $\mathbb{Y}$;

(vi) if $x_{1} \in x_{2}+\mathbb{K}$, then $\xi_{e}\left(x_{2}\right) \leq \xi_{e}\left(x_{1}\right)$;

(vii) $\xi_{e}\left(x_{1}+x_{2}\right) \leq \xi_{e}\left(x_{1}\right)+\xi_{e}\left(x_{2}\right)$ for all $x_{1}, x_{2} \in \mathbb{Y}$.

Proof See, for instance, [7, 20-23].

The following result is Theorem 2.1 of $\mathrm{Du}$ [24].

Proposition 2.5 Let $\left(\mathbb{X},\left\{d_{\alpha}\right\}_{\alpha}\right)$ be a cone pseudo-metric space over a solid cone $\mathbb{K}$. Then the family of mappings $\delta_{\alpha}: \mathbb{X} \times \mathbb{X} \rightarrow\left[0, \infty\left[\right.\right.$ defined by $\delta_{\alpha}=\xi_{e} \circ d_{\alpha}$ is a family of pseudo-metrics on $\mathbb{X}$.

Proof Since $\xi_{e}(\cdot)$ is a seminorm on $\mathbb{Y}$ by Lemma 2.4, Proposition 2.3 gives the result.

If the cone $\mathbb{K}$ is normal and solid, then $\xi_{e}(\cdot)$ is a norm over $\mathbb{Y}$, and we have the following proposition. 
Proposition 2.6 If $(\mathbb{Y}, \tau)$ is a Hausdorff topological space ordered by a normal solid cone $\mathbb{K}$, then $(\mathbb{Y}, \tau)$ is a normable space.

Proof See Proposition 1.10 in [18], Chapter 2.

Next, we discuss some convergence properties of cone pseudo-metric spaces. We note that $x \ll y$ if and only if $y-x \in \operatorname{int} \mathbb{K}$, where the 'int' is the interior.

Definition 2.7 Let $\left(\mathbb{X},\left\{d_{\alpha}\right\}_{\alpha}\right)$ be a cone pseudo-metric space over a solid convex cone $\mathbb{K} \subset \mathbb{Y}$, where $\mathbb{Y}$ is a locally convex space, $x \in \mathbb{X}$, and $\left\{x_{n}\right\}_{n}$ a sequence in $\mathbb{X}$.

1. $\left\{x_{n}\right\}_{n}$ is Cauchy sequence whenever for every $\alpha \in \Lambda$ and $c \in \mathbb{Y}$ with $\theta \ll c$, there is a natural number $N_{0}$ such that

$$
d_{\alpha}\left(x_{n}, x_{m}\right) \ll c, \quad \forall n, m \geq N_{0} .
$$

2. $\left\{x_{n}\right\}_{n}$ converges to $x$ whenever for every $\alpha \in \Lambda$ and $c \in \mathbb{Y}$ with $\theta \ll c$, there is a natural number $N_{0}$ such that

$$
d_{\alpha}\left(x_{n}, x\right) \ll c, \quad \forall n \geq N_{0} .
$$

3. ( $\left.\mathbb{X},\left\{d_{\alpha}\right\}_{\alpha}\right)$ is complete if each Cauchy sequence converges in $\mathbb{X}$.

Proposition 2.8 Let $\left(\mathbb{X},\left\{d_{\alpha}\right\}_{\alpha}\right)$ be a cone pseudo-metric space over a solid convex cone $\mathbb{K} \subset \mathbb{Y}$, where $\mathbb{Y}$ is a locally convex space.

Then, for each $\alpha \in \Lambda$, we get

$$
d_{\alpha}\left(x_{n}, x\right) \longrightarrow \theta \quad \Longleftrightarrow \quad \delta_{\alpha}\left(x_{n}, x\right)=\xi_{e}\left(d_{\alpha}\left(x_{n}, x\right)\right) \longrightarrow 0
$$

Proof It is similar to the proof of Theorem 3.2 in [25].

Using this pseudo-metric $\delta_{\alpha}$, we keep saying that $\left(\mathbb{X},\left\{\delta_{\alpha}\right\}_{\alpha}\right)$ is a pseudo-metric space over a solid convex cone $\mathbb{K}$.

\section{Fixed point theorems}

Recall that the most famous ordering principle.

Theorem 3.1 (Brezis-Browder) Let ( $W, \precsim$ ) be a quasi-ordered set (i.e. $\precsim$ is a reflexive and transitive relation), and let $\Psi: W \longrightarrow \mathbb{R}$ be a function satisfying the following conditions:

(B1) $\Psi$ is bounded below;

(B2) $w_{1} \precsim w_{2} \Longrightarrow \Psi\left(w_{1}\right) \leq \Psi\left(w_{2}\right)$;

(B3) For every decreasing sequence $\left\{w_{n}\right\}_{n \in \mathbb{N}} \subset W$ with respect to ' $\precsim$ ', there exists $w \in W$ such that $w \leq w_{n}$ for all $n \in \mathbb{N}$.

Then, for every $w_{0} \in W$, there exists $\bar{w} \in W$ such that

(i) $\bar{w} \precsim w_{0}$;

(ii) $\hat{w} \precsim \bar{w} \Longrightarrow \Psi(\hat{w})=\Psi(\bar{w})$. 
In particular, if we strengthen (B2) to

$\left(\mathrm{B} 2^{\prime}\right) \quad\left(w_{1} \precsim w_{2}, w_{1} \neq w_{2}\right) \Longrightarrow \Psi\left(w_{1}\right)<\Psi\left(w_{2}\right)$,

then

(ii') $\hat{w} \precsim \bar{w} \Longrightarrow \hat{w}=\bar{w}$, that is, $\bar{w}$ is minimal in $W$ with respect to '

Proof See Corollary 1 in [8].

Now we are able to give the main result of this section.

Theorem 3.2 Let $\left(\mathbb{Y},\left\{p_{i}\right\}_{i \in I}\right)$ a complete separated locally convex space, $\left(\mathbb{X},\left\{\delta_{\alpha}\right\}_{\alpha \in \Lambda}\right)$ be a complete Hausdorff pseudo-metric space over a solid convex cone $\mathbb{K}, T: \mathbb{X} \longrightarrow 2^{\mathbb{X}}$ and $S: \mathbb{X} \longrightarrow 2^{\mathbb{Y}}$ two set-valued maps with nonempty values.

Suppose that, for every $(\alpha, i) \in \Lambda \times I$ and two constants $c_{\alpha}, c_{i}>0$, there exist lower semicontinuous functions $\varphi_{\alpha i}: \mathbb{Y} \longrightarrow[0, \infty)$, and for each $(x, y) \in G_{S}$, there exist $u \in T x$ and $v \in$ Su such that

$$
\max \left\{c_{\alpha} \delta_{\alpha}(x, u), c_{i} p_{i}(y-v)\right\} \leq \varphi_{\alpha i}(y)-\varphi_{\alpha i}(v)
$$

Then $T$ has a fixed point in $\mathbb{X}$.

Proof Put

$$
W_{0}=\left\{(x, y) \in G_{S} ; \forall(\alpha, i) \in \Lambda \times I, \max \left\{c_{\alpha} \delta_{\alpha}\left(x_{0}, x\right), c_{i} p_{i}\left(y_{0}-y\right)\right\}+\varphi_{\alpha i}(y) \leq \varphi_{\alpha i}\left(y_{0}\right)\right\}
$$

for some $\left(x_{0}, y_{0}\right) \in G_{S}$. Then $W_{0}$ is a nonempty closed subset of $G_{S}$. Indeed, let $\left(x_{n}, y_{n}\right)_{n}$ be a sequence in $W_{0}$ that converges to $(x, y)$, that is, $\lim _{n \rightarrow \infty} p_{i}\left(y_{n}-y\right)=0$. Since for each $(\alpha, i) \in \Lambda \times I$, the function $\varphi_{\alpha i}$ is lower semicontinuous, that is,

$$
\varphi_{\alpha i}(y) \leq \liminf _{n \rightarrow \infty} \varphi_{\alpha i}\left(y_{n}\right)
$$

we have

$$
\begin{aligned}
c_{i} p_{i}\left(y_{0}-y\right) & \leq c_{i} p_{i}\left(y_{0}-y_{n}\right)+c_{i} p_{i}\left(y_{n}-y\right) \\
& \leq \varphi_{\alpha i}\left(y_{0}\right)-\varphi_{\alpha i}\left(y_{n}\right)+c_{i} p_{i}\left(y_{n}-y\right) \\
& \leq \varphi_{\alpha i}\left(y_{0}\right)-\liminf _{k \rightarrow \infty} \varphi_{\alpha i}\left(y_{k}\right)+c_{i} p_{i}\left(y_{n}-y\right) \\
& \leq \varphi_{\alpha i}\left(y_{0}\right)-\varphi_{\alpha i}(y)+c_{i} p_{i}\left(y_{n}-y\right) .
\end{aligned}
$$

So, taking the limit with respect to $n$, we get $c_{i} p_{i}\left(y_{0}-y\right) \leq \varphi_{\alpha i}\left(y_{0}\right)-\varphi_{\alpha i}(y)$, and by similar arguments we get

$$
c_{\alpha} \delta_{\alpha}\left(x_{0}, x\right) \leq \varphi_{\alpha i}\left(y_{0}\right)-\varphi_{\alpha i}(y)
$$

Hence, $\max \left\{c_{\alpha} \delta_{\alpha}\left(x_{0}, x\right), c_{i} p_{i}\left(y_{0}-y\right)\right\}+\varphi_{\alpha i}(y) \leq \varphi_{\alpha i}\left(y_{0}\right)$, so that $(x, y) \in W_{0}$. 
Now we define a binary relation in $W_{0}$ as follows: for every $\left(x_{1}, y_{1}\right)$ and $\left(x_{2}, y_{2}\right)$ in $W_{0}$,

$$
\left(x_{1}, y_{1}\right) \precsim\left(x_{2}, y_{2}\right) \Longleftrightarrow \max \left\{c_{\alpha} \delta_{\alpha}\left(x_{1}, x_{2}\right), c_{i} p_{i}\left(y_{1}-y_{2}\right)\right\} \leq \varphi_{\alpha i}\left(y_{2}\right)-\varphi_{\alpha i}\left(y_{1}\right)
$$

for each $(\alpha, i) \in \Lambda \times I$. We can show that the relation $\precsim$ is an ordering on $W_{0}$.

Next, we show that, for every decreasing sequence $\left(x_{n}, y_{n}\right)_{n \in \mathbb{N}} \subset W_{0}$ with respect to ' $\precsim$ ', there exists $\left(x^{*}, y^{*}\right) \in W_{0}$ such that $\left(x^{*}, y^{*}\right) \precsim\left(x_{n}, y_{n}\right)$ for all $n \in \mathbb{N}$. Let $\left(x_{n}, y_{n}\right)_{n \in \mathbb{N}}$ be a decreasing sequence in $W_{0}$. Then, for any $m, n \in \mathbb{N}$ such that $m \geq n$, we have

$$
\left(x_{m}, y_{m}\right) \precsim\left(x_{n}, y_{n}\right) \Longleftrightarrow \max \left\{c_{\alpha} \delta_{\alpha}\left(x_{m}, x_{n}\right), c_{i} p_{i}\left(y_{m}-y_{n}\right)\right\} \leq \varphi_{\alpha i}\left(y_{n}\right)-\varphi_{\alpha i}\left(y_{m}\right)
$$

for each $(\alpha, i) \in \Lambda \times I$,

which gives that the positive sequence $\left\{\varphi_{\alpha i}\left(y_{n}\right)\right\}_{n}$ is decreasing (for $\alpha$ and $i$ fixed). Hence, there exists $r_{\alpha i}$ such that $\lim \varphi_{\alpha i}\left(y_{n}\right)=r_{\alpha i}$. Let $\varepsilon>0$ and $(\alpha, i) \in \Lambda \times I$. There exists $N_{0} \in \mathbb{N}^{*}$ such that, for any $n \geq N_{0}$, we have

$$
r_{\alpha i} \leq \varphi_{\alpha i}\left(y_{n}\right) \leq r_{\alpha i}+\min \left(c_{\alpha}, c_{i}\right) \cdot \varepsilon
$$

and then, for every $m \geq n \geq N_{0}$,

$$
\begin{aligned}
c_{i} p_{i}\left(y_{m}-y_{n}\right) & \leq \varphi_{\alpha i}\left(y_{n}\right)-\varphi_{\alpha i}\left(y_{m}\right) \\
& \leq r_{\alpha i}+\min \left(c_{\alpha}, c_{i}\right) \cdot \varepsilon-r_{\alpha i} .
\end{aligned}
$$

Thus,

$$
c_{i} p_{i}\left(y_{m}-y_{n}\right) \leq \min \left(c_{\alpha}, c_{i}\right) \cdot \varepsilon \leq c_{i} \varepsilon .
$$

Also, we get

$$
\begin{aligned}
c_{\alpha} \delta_{\alpha}\left(x_{m}, x_{n}\right) & \leq \varphi_{\alpha i}\left(y_{n}\right)-\varphi_{\alpha i}\left(y_{m}\right) \\
& \leq r_{\alpha i}+\min \left(c_{\alpha}, c_{i}\right) \cdot \varepsilon-r_{\alpha i}
\end{aligned}
$$

and thus

$$
c_{\alpha} \delta_{\alpha}\left(x_{m}, x_{n}\right) \leq c_{\alpha} \varepsilon .
$$

Repeating the last computation for every $(\alpha, i) \in \Lambda \times I$ and using the fact that $\left\{\delta_{\alpha}\right\}_{\alpha \in \Lambda}$ and $\left\{p_{i}\right\}_{i \in I}$ are separated families, we obtain that $\left\{x_{n}\right\}_{n}$ and $\left\{y_{n}\right\}_{n}$ are Cauchy sequences in the complete spaces $\mathbb{X}$ and $\mathbb{Y}$, respectively. Therefore, there exist $x^{*} \in \mathbb{X}$ and $y^{*} \in \mathbb{Y}$ such that

$$
x_{n} \longrightarrow x^{*} \text { and } y_{n} \longrightarrow y^{*} \text {. }
$$

Since $W_{0}$ is closed, we have that $\left(x^{*}, y^{*}\right) \in W_{0}$ and $y^{*} \in S x^{*}$ by the definition of $W_{0}$. 
Also, for all $(n, m) \in \mathbb{N}^{2}$ such that $m \geq n$, we have $\left(x_{m}, y_{m}\right) \precsim\left(x_{n}, y_{n}\right)$, so that for all $(\alpha, i) \in$ $\Lambda \times I$,

$$
\begin{aligned}
\max \left\{c_{\alpha} \delta_{\alpha}\left(x_{m}, x_{n}\right), c_{i} p_{i}\left(y_{m}-y_{n}\right)\right\} & \leq \varphi_{\alpha i}\left(y_{n}\right)-\varphi_{\alpha i}\left(y_{m}\right) \\
& \leq \varphi_{\alpha i}\left(y_{n}\right)-\liminf _{k \rightarrow \infty} \varphi_{\alpha i}\left(y_{k}\right) \\
& \leq \varphi_{\alpha i}\left(y_{n}\right)-\varphi_{\alpha i}\left(y^{*}\right) .
\end{aligned}
$$

Taking the limit with respect to $m$ and using the fact that $\delta_{\alpha}$ and $p_{i}$ are continuous, we get

$$
\max \left\{c_{\alpha} \delta_{\alpha}\left(x^{*}, x_{n}\right), c_{i} p_{i}\left(y^{*}-y_{n}\right)\right\} \leq \varphi_{\alpha i}\left(y_{n}\right)-\varphi_{\alpha i}\left(y^{*}\right) \quad \text { for all }(\alpha, i) \in \Lambda \times I .
$$

Thus, for each $n \in \mathbb{N}$,

$$
\left(x^{*}, y^{*}\right) \precsim\left(x_{n}, y_{n}\right) .
$$

Let $(\alpha, i) \in \Lambda \times I$ be fixed and choose $\Psi: W_{0} \longrightarrow \mathbb{R}$ as follows: $\Psi(x, y)=\varphi_{\alpha i}(y)$ for each $(x, y) \in W_{0}$. Condition (B1) from Theorem 3.1 holds since $\varphi_{\alpha i}(y) \geq 0$. We also have

$$
\left(x_{1}, y_{1}\right) \precsim\left(x_{2}, y_{2}\right) \quad \Longrightarrow \quad \varphi_{\alpha i}\left(y_{1}\right) \leq \varphi_{\alpha i}\left(y_{2}\right) \quad \text { for each }(\alpha, i) \in \Lambda \times I \text {. }
$$

So $\Psi\left(x_{1}, y_{1}\right) \leq \Psi\left(x_{2}, y_{2}\right)$, and thus (B2) also holds. Then all assumptions of the BrezisBrowder principle are satisfied. Hence, for each $\left(x_{0}, y_{0}\right) \in W_{0}$, there exists $(\bar{x}, \bar{y}) \in W_{0}$ such that:

(i) $(\bar{x}, \bar{y}) \precsim\left(x_{0}, y_{0}\right)$;

(ii) if $(\hat{x}, \hat{y}) \precsim(\bar{x}, \bar{y})$, then $\Psi(\hat{x}, \hat{y})=\Psi(\bar{x}, \bar{y})$.

We claim that $\bar{x}$ is a fixed point for $T$. For this $(\bar{x}, \bar{y}) \in W_{0} \subset G_{S}$, there exists $(u, v) \in \mathbb{X} \times \mathbb{Y}$ such that $u \in T \bar{x}$ and $v \in S \bar{u}$ satisfy the following inequality for each $(\alpha, i) \in \Lambda \times I$ :

$$
\max \left\{c_{\alpha} \delta_{\alpha}(u, \bar{x}), c_{i} p_{i}(v-\bar{y})\right\} \leq \varphi_{\alpha i}(\bar{y})-\varphi_{\alpha i}(v)
$$

Given $(u, v) \precsim(\bar{x}, \bar{y})$, we have $\Psi(u, v)=\Psi(\bar{x}, \bar{y})$; hence, $x=\bar{x}$, and thus $\bar{x} \in T \bar{x}$, which completes the proof.

Theorem 3.3 Under the hypotheses of Theorem 3.2, suppose that the condition 'for each $(x, y) \in G_{S}$, there exist $u \in T x$ and $v \in S u$ ' is replaced by 'for each $(x, y) \in G_{S}$ and for every $u \in T x$, there exists $v \in S u$ ?

Then $T$ has a critical point, that is, there exists $\bar{x} \in \mathbb{X}$ such that $\{\bar{x}\}=T \bar{x}$.

Proof By Theorem 3.2, $T$ has a fixed point $\bar{x}$ in $\mathbb{X}$. We claim that it is a critical point. For this, let us show that assumption (B2') of Brezis-Browder holds, and so we have (ii'). Let $(\alpha, i) \in \Lambda \times I$ be fixed and choose $\Psi: W_{0} \longrightarrow \mathbb{R}$ as in the above proof: $\Psi(x, y)=\varphi_{\alpha i}(y)$ for each $(x, y) \in W_{0}$. Then

$$
\left(x_{1}, y_{1}\right) \precsim\left(x_{2}, y_{2}\right), \quad\left(x_{1}, y_{1}\right) \neq\left(x_{2}, y_{2}\right) \quad \Longrightarrow \quad \Psi\left(x_{1}, y_{1}\right)<\Psi\left(x_{2}, y_{2}\right) .
$$


Indeed, suppose that $x_{1} \neq x_{2}$. Then, for each $\alpha \in \Lambda$, we get

$$
\delta_{\alpha}\left(x_{1}, x_{2}\right) \neq 0 \quad \Longrightarrow \quad \delta_{\alpha}\left(x_{1}, x_{2}\right)>0
$$

Then

$$
0<c_{\alpha} \delta_{\alpha}\left(x_{1}, x_{2}\right) \leq \varphi_{\alpha i}\left(y_{2}\right)-\varphi_{\alpha i}\left(y_{1}\right)
$$

and hence $\varphi_{\alpha i}\left(y_{1}\right)<\varphi_{\alpha i}\left(y_{2}\right) \Longleftrightarrow \Psi\left(x_{1}, y_{1}\right)<\Psi\left(x_{2}, y_{2}\right)$.

Otherwise, if $x_{1}=x_{2}$, then by the assumption $\left(x_{1}, y_{1}\right) \neq\left(x_{2}, y_{2}\right)$ we must have $y_{1} \neq y_{2}$, and then $\varphi_{\alpha i}\left(y_{1}\right)<\varphi_{\alpha i}\left(y_{2}\right)$. Therefore, assumption (B2') in Theorem 3.1 is satisfied. Then $(\bar{x}, \bar{y})$ is minimal point in $W_{0}$ by (ii') of the Brezis-Browder principle.

Now we claim that $\bar{x}$ is a critical point for $T$. By inequality (1) we have

$$
\max \left\{c_{\alpha} \delta_{\alpha}(u, \bar{x}), c_{i} p_{i}(v-\bar{y})\right\} \leq \varphi_{\alpha i}(\bar{y})-\varphi_{\alpha i}(v)
$$

for each $u \in T \bar{x}$ and $(\alpha, i) \in \Lambda \times I$, and then $(u, v) \precsim(\bar{x}, \bar{y})$. Since $(\bar{x}, \bar{y})$ is a minimal point in $W_{0}$, it follows that $u=\bar{x}$, and thus $T \bar{x}=\{\bar{x}\}$, which completes the proof.

By the same process as before we can also get the same results if we replace the cone pseudo-distance $\left\{\delta_{\alpha}\right\}_{\alpha \in \Lambda}$ with respect to the solid cone with the real-valued pseudodistance $\left\{d_{\alpha}\right\}_{\alpha \in \Lambda}$.

Proposition 3.4 Let $\left(\mathbb{X},\left\{d_{\alpha}\right\}_{\alpha \in \Lambda}\right)$ be a complete Hausdorff pseudo-metric space, $\left(\mathbb{Y},\left\{p_{i}\right\}_{i \in I}\right)$ a complete separated locally convex space, and $T: \mathbb{X} \longrightarrow 2^{\mathbb{X}}$ and $S: \mathbb{X} \longrightarrow 2^{\mathbb{Y}}$ two setvalued maps with nonempty values.

Suppose that, for every $(\alpha, i) \in \Lambda \times I$ and two constants $c_{\alpha}, c_{i}>0$, there exist lower semicontinuous functions $\varphi_{\alpha i}: \mathbb{Y} \longrightarrow[0, \infty)$ and, for each $(x, y) \in G_{S}$, there exist $u \in T x$ and $v \in S u$ (resp., for every $u \in T x$, there exists $v \in S u$ ) such that:

$$
\max \left\{c_{\alpha} d_{\alpha}(x, u), c_{i} p_{i}(y-v)\right\} \leq \varphi_{\alpha i}(y)-\varphi_{\alpha i}(v)
$$

Then $T$ has a fixed point (resp. critical point) in $\mathbb{X}$.

If the set-valued map $S$ in Proposition 3.4 is only a single-valued map, then we have the following:

Corollary 3.5 (Isac [12]) Let ( $\left.\mathbb{X},\left\{p_{\alpha}\right\}_{\alpha \in \Lambda}\right)$ be a Hausdorff locally convex space, and $M \subset \mathbb{X}$ be a nonempty set. The set-valued map $T: \mathbb{X} \longrightarrow 2^{\mathbb{X}}$ has a critical point if and only if there exist a complete Hausdorff locally convex space $\left(\mathbb{Y},\left\{q_{i}\right\}_{i \in I}\right)$, a subset $M_{0} \subseteq M, S: M_{0} \longrightarrow \mathbb{Y}$, for every couple $(\alpha, i) \in \Lambda \times I$, a function $\varphi_{\alpha i}: \overline{S\left(M_{0}\right)} \longrightarrow[0, \infty)$, and two constants $c_{\alpha}, c_{i}>0$ such that:

(i) $T\left(M_{0}\right) \subset M_{0}$, and $M_{0} \subset M$ is closed;

(ii) $S$ is closed, and $\overline{S\left(M_{0}\right)}$ is complete;

(iii) $\varphi_{\alpha i}$ is lower semicontinuous for each $(\alpha, i) \in \Lambda \times I$;

(iv) $\max \left\{c_{\alpha} p_{\alpha}(x-y), c_{i} q_{i}(S(x)-S(y))\right\} \leq \varphi_{\alpha i}(S(x))-\varphi_{\alpha i}(S(y))$ for all $x \in M_{0}$ and all $y \in T x$. 
Proof If $T$ has a critical point $\bar{x} \in M$, then the assumptions of Isac's theorem are satisfied if we put $M_{0}=\{\bar{x}\}, \mathbb{X}=\mathbb{Y},\left\{p_{\alpha}\right\}_{\alpha \in \Lambda}=\left\{q_{i}\right\}_{i \in I}, S=I_{M_{0}}$, and for each $(\alpha, i) \in \Lambda \times I, c_{\alpha}=c_{i}=1$ and $\varphi_{\alpha i}=0$.

Conversely, $\left\{p_{\alpha}\right\}_{\alpha \in \Lambda}$ is generating family of separated seminorms on $\mathbb{X}$, and if we set

$$
p_{\alpha}(x-y)=d_{\alpha}(x, y)
$$

for each $\alpha \in \Lambda$, then $\left(M_{0},\left\{d_{\alpha}\right\}_{\alpha \in \Lambda}\right)$ is a complete Hausdorff pseudo-metric subspace of $\mathbb{X}$. Also, by (ii) we get that $\left(\overline{S\left(M_{0}\right)},\left\{q_{i}\right\}_{i \in I}\right)$ is a complete Hausdorff locally convex subspace of $\mathbb{Y}$, and since $T\left(M_{0}\right) \subset M_{0}$, all assumptions of Proposition 3.4 are satisfied, so that we get the result.

Remark 3.6 Our main result does not involve any assumptions about closeness of intermediary set-valued map $S$, contrary to the result of Isac [12].

Corollary 3.7 (Fang [11]) Let $T: \mathbb{X} \longrightarrow \mathbb{X}$ be a map of a complete Hausdorfflocally convex space $\left(\mathbb{X},\left\{p_{\alpha}\right\}_{\alpha \in \Lambda}\right)$. Suppose that there exists a lower semicontinuous function $\varphi: \mathbb{X} \longrightarrow$ $[0, \infty)$ such that, for each $x \in \mathbb{X}$ and for each $\alpha \in \Lambda$,

$$
p_{\alpha}(x-T x) \leq \varphi(x)-\varphi(T x)
$$

Then T has a fixed point.

Proof For every $x, y \in \mathbb{X}$, we even replace $p_{\alpha}(x-y)=d_{\alpha}(x, y)$ and take single-valued maps $T^{\prime}$ and $S$ with $S x=\{x\}$ and $T^{\prime} x=\{T x\}$ for all $x \in \mathbb{X}$. Then inequality (3) implies inequality (2) of Proposition 3.4, and the result follows.

We get the next obvious two corollaries.

Corollary 3.8 (Downing and Kirk [15]) Let $\mathbb{X}$ and $\mathbb{Y}$ be complete metric spaces, and $T$ : $\mathbb{X} \rightarrow \mathbb{X}$ an arbitrary mapping. Suppose that there exist a closed mapping $S: \mathbb{X} \longrightarrow \mathbb{Y}, a$ lower semicontinuous mapping $\varphi: S(\mathbb{X}) \longrightarrow[0, \infty)$, and a constant $c>0$ such that, for each $x \in \mathbb{X}$,

$$
\max \left\{d_{\mathbb{X}}(x, T x), c d_{\mathbb{Y}}(S(x), S(T x))\right\} \leq \varphi(S(x))-\varphi(S(T x))
$$

Then there exists $x \in \mathbb{X}$ such that $T x=x$.

Corollary 3.9 (Caristi $[14])$ Let $(\mathbb{X}, d)$ be a complete metric space, and let $\varphi: \mathbb{X} \longrightarrow[0, \infty)$ be a lower semicontinuous function. If a mapping $T: \mathbb{X} \longrightarrow \mathbb{X}$ satisfies for each $x \in \mathbb{X}$ the condition

$$
d(x, T x) \leq \varphi(x)-\varphi(T x)
$$

then $T$ has a fixed point in $\mathbb{X}$.

We conclude this section with an application of Theorem 3.2. 
Theorem 3.10 Let $\left(\mathbb{Y},\left\{p_{i}\right\}_{i \in I}\right)$ be a complete separated locally convex space, $\left(\mathbb{X},\left\{\delta_{\alpha}\right\}_{\alpha \in \Lambda}\right)$ be a complete Hausdorff pseudo-metric space over a solid cone $\mathbb{K}, S: \mathbb{X} \longrightarrow 2^{\mathbb{Y}}$ be set-valued map, and for every $(\alpha, i) \in \Lambda \times I, \varphi_{\alpha i}: \mathbb{Y} \longrightarrow[0, \infty)$ be lower semicontinuous function.

Suppose that, for each $(x, y) \in G_{S}$, there exists $\left(x_{0}, y_{0}\right) \in G_{S}$ such that

1. $x_{0} \neq x$;

2. $\varphi_{\alpha i}\left(y_{0}\right)+\max \left\{c_{\alpha} \delta_{\alpha}\left(x, x_{0}\right), c_{i} p_{i}\left(y-y_{0}\right)\right\} \leq \varphi_{\alpha i}(y)$ for every $(\alpha, i) \in \Lambda \times I$.

Then there exist $(\bar{x}, \bar{y}) \in G_{S}$ and $\left(\alpha_{0}, i_{0}\right) \in \Lambda \times I$ such that $\varphi_{\alpha_{0} i_{0}}(\bar{y})=\inf _{t \in \mathbb{Y}} \varphi_{\alpha_{0} i_{0}}(t)$.

Proof By contradiction suppose that, for each $(x, y) \in G_{S}$ and for every $(\alpha, i) \in \Lambda \times I$, we have

$$
\varphi_{\alpha i}(y)>\inf _{t \in \mathbb{Y}} \varphi_{\alpha i}(t)
$$

By assumptions, there exists $\left(x_{0}, y_{0}\right) \in G_{S}$ such that 1 and 2 hold. Set

$$
\begin{aligned}
E(x, y)= & \left\{(z, t) \in G_{S}: z \neq x, \text { and } \forall(\alpha, i) \in \Lambda \times I,\right. \\
& \left.\varphi_{\alpha i}(t)+\max \left\{c_{\alpha} \delta_{\alpha}(x, z), c_{i} p_{i}(y-t)\right\} \leq \varphi_{\alpha i}(y)\right\} .
\end{aligned}
$$

For all $(x, y) \in G_{S}$, we have $\left(x_{0}, y_{0}\right) \in E(x, y)$ and $(x, y) \notin E(x, y)$. For all $x \in \mathbb{X}$, we put $G_{S}(x)=$ $\left\{y \in \mathbb{Y}:(x, y) \in G_{S}\right\}$. Define the set-valued map $T$ by

$$
T x=\bigcup_{y \in G_{S}(x)}\{z \in \mathbb{X}: \exists t \in S z \text { such that }(z, t) \in E(x, y)\}
$$

for $x \in \mathbb{X}$. For all $(x, y) \in G_{S}$ and $(\alpha, i) \in \Lambda \times I$, there exist $z \in T x$ and $t \in S z$ such that

$$
\max \left\{c_{\alpha} \delta_{\alpha}(x, z), c_{i} p_{i}(y-t)\right\} \leq \varphi_{\alpha i}(y)-\varphi_{\alpha i}(t)
$$

Then by Theorem 3.2, T admits a point $\bar{x}$ such that $\bar{x} \in T \bar{x}$. For this $\bar{x}$, we get that, for some $\bar{y}_{1}, \bar{y}_{2} \in \mathbb{Y},\left(\bar{x}, \bar{y}_{1}\right) \in E\left(\bar{x}, \bar{y}_{2}\right)$, which is absurd.

\section{Variational principle}

Theorem 4.1 Let $\left(\mathbb{Y},\left\{p_{i}\right\}_{i \in I}\right)$ be a complete separated locally convex space, $\left(\mathbb{X},\left\{\delta_{\alpha}\right\}_{\alpha \in \Lambda}\right)$ be a complete Hausdorff pseudo-metric space over a solid cone $\mathbb{K}, S: \mathbb{X} \longrightarrow 2^{\mathbb{Y}}$ be a set-valued map, and, for every $(\alpha, i) \in \Lambda \times I, \varphi_{\alpha i}: \mathbb{Y} \longrightarrow[0, \infty)$ be a lower semicontinuous function.

Then, for each $\varepsilon>0$ and $\left(x_{0}, y_{0}\right) \in G_{S}$ satisfying

$$
\varphi_{\alpha i}\left(y_{0}\right) \leq \inf \varphi_{\alpha i}+\varepsilon, \quad \forall(\alpha, i) \in \Lambda \times I
$$

there exists $(\bar{x}, \bar{y}) \in G_{S}$ such that:

(i) for each $(\alpha, i) \in \Lambda \times I, \varphi_{\alpha i}(\bar{y}) \leq \varphi_{\alpha i}\left(y_{0}\right)$;

(ii) for each $(x, y) \in G_{S}$ with $x \neq \bar{x}$, there exist $(\alpha, i) \in \Lambda \times I$ and two constants $c_{\alpha}, c_{i}>0$ such that

$$
\varphi_{\alpha i}(\bar{y})<\varphi_{\alpha i}(y)+\varepsilon \max \left\{c_{\alpha} \delta_{\alpha}(x, \bar{x}), c_{i} p_{i}(y-\bar{y})\right\} .
$$


Proof Let $\varepsilon>0$ and $\left(x_{0}, y_{0}\right) \in G_{S}$. Put

$$
W_{0}=\left\{(x, y) \in G_{S} ; \forall(\alpha, i) \in \Lambda \times I, \varphi_{\alpha i}(y)+\varepsilon \max \left\{c_{\alpha} \delta_{\alpha}\left(x, x_{0}\right), c_{i} p_{i}\left(y-y_{0}\right)\right\} \leq \varphi_{\alpha i}\left(y_{0}\right)\right\}
$$

It is a nonempty and closed subset of $G_{S}$ since the family $\left\{\varphi_{\alpha i}\right\}_{\alpha i}$ is lower semicontinuous.

For all $x \in \mathbb{X}$, we put $W_{0}(x)=\left\{y \in \mathbb{Y}:(x, y) \in W_{0}\right\}$. Next, we define the set-valued map $T: \mathbb{X} \longrightarrow 2^{\mathbb{X}}$ by

$$
\begin{aligned}
T x= & \bigcup_{y \in W_{0}(x)}\{\hat{x} \in \mathbb{X} ; \exists \hat{y} \in S \hat{x}, \forall(\alpha, i) \in \Lambda \times I, \\
& \left.\varphi_{\alpha i}(\hat{y})+\varepsilon \max \left\{c_{\alpha} \delta_{\alpha}(\hat{x}, x), c_{i} p_{i}(\hat{y}-y)\right\} \leq \varphi_{\alpha i}(y)\right\} .
\end{aligned}
$$

Obviously, $T$ satisfies inequality (1) of Theorem 3.2 with $\phi_{\alpha i}=\frac{1}{\varepsilon} \varphi_{\alpha i}$ so that $T$ has a fixed point, that is, there exists $(\bar{x}, \bar{y}) \in W_{0}$ such that $\bar{x} \in T \bar{x}$ with

$$
(\bar{x}, \bar{y}) \in W_{0} \quad \Longrightarrow \quad \varphi_{\alpha i}(\bar{y}) \leq \varphi_{\alpha i}\left(y_{0}\right)
$$

and if $(\hat{x}, \hat{y}) \in G_{S}$ with $(\hat{x}, \hat{y}) \precsim(\bar{x}, \bar{y})$, then $\hat{x}=\bar{x}$, which is equivalent to the assertion that, for each $(x, y) \in G_{S}$ with $x \neq \bar{x}$, there exist $(\alpha, i) \in \Lambda \times I$ and two constants $c_{\alpha}, c_{i}>0$ such that

$$
\varphi_{\alpha i}(\bar{y})<\varphi_{\alpha i}(y)+\varepsilon \max \left\{c_{\alpha} \delta_{\alpha}(x, \bar{x}), c_{i} p_{i}(y-\bar{y})\right\} .
$$

The proof is complete.

Remark 4.2 We claim that Theorem 4.1 implies Theorem 3.2. Indeed, let $\left(x_{0}, y_{0}\right) \in G_{S}$ be given and take $\varepsilon=1$. By Theorem 4.1 there exists $(\bar{x}, \bar{y}) \in G_{S}$ such that assertions (i) and (ii) hold. Since (i), we have $(\bar{x}, \bar{y}) \in W_{0}$. We claim that $\bar{x}$ is a fixed point of $T$. Assuming the contrary, by inequality (1) we get the existence of some $(x, y) \in G_{S}$ such that $x \in T \bar{x}, x \neq \bar{x}$, and

$$
\max \left\{c_{\alpha} \delta_{\alpha}(x, \bar{x}), c_{i} p_{i}(y-\bar{y})\right\} \leq \varphi_{\alpha i}(\bar{y})-\varphi_{\alpha i}(y) \quad \text { for every }(\alpha, i) \in \Lambda \times I
$$

This contradicts (ii). Hence, $\bar{x}$ is a fixed point.

The above considerations show that Theorem 4.1 and Theorem 3.2 are equivalent.

Since the Caristi theorem (Corollary 3.9) is a particular case of our main result and the Ekeland variational principle is equivalent to Caristi's theorem, Theorem 4.1 is a generalization of the variational principle of Ekeland:

Corollary 4.3 (Ekeland [1]) Let $(\mathbb{X}, d)$ be a complete metric space, and $\varphi: \mathbb{X} \longrightarrow[0, \infty)$ bea lower semicontinuous function. Let $\varepsilon>0$, and let a point $u \in \mathbb{X}$ be such that $\varphi(u) \leq \inf \varphi+\varepsilon$. Then there exists a point $v \in \mathbb{X}$ such that:

(i) $\varphi(v) \leq \varphi(u)$;

(ii) $\varphi(v)<\varphi(w)+\varepsilon d(w ; v)$ for any $w \in \mathbb{X} ; w \neq v$. 


\section{Applications}

In this section, we propose two applications.

\subsection{General nonlinear complementarity problem}

In a Hilbert space $(\mathbb{X},\langle\cdot, \cdot\rangle)$, the dual cone $\mathbb{K}^{\prime}$ of a convex cone $\mathbb{K}$ with respect to the duality $\left\langle\mathbb{X}^{\prime}, \mathbb{X}\right\rangle$ is defined by

$$
\mathbb{K}^{\prime}=\{y \in \mathbb{X}:\langle y, x\rangle \geq 0, \forall x \in \mathbb{K}\}
$$

and the polar of $\mathbb{K}$ is $\mathbb{K}^{0}=-\mathbb{K}^{\prime}$.

Next, we suppose that $\mathbb{K}$ is a closed convex cone in $\mathbb{X}$. It is shown in [26] that the projection operator onto $\mathbb{K}$, denoted by $P_{\mathbb{K}}$, is well defined and satisfies, for all $x \in \mathbb{X}$,

$$
\left\|x-P_{\mathbb{K}}(x)\right\|=\min _{y \in \mathbb{K}}\|x-y\| .
$$

The next two results can be found in [26].

Theorem 5.1 For every $x \in \mathbb{X}, P_{\mathbb{K}}$ has the following properties:

1. $\left\langle P_{\mathbb{K}}(x)-x, y\right\rangle \geq 0$ for every $y \in \mathbb{K}$;

2. $\left\langle P_{\mathbb{K}}(x)-x, P_{\mathbb{K}}(x)\right\rangle=0$.

Theorem 5.2 For all $x, y, z \in \mathbb{X}$, the following statements are equivalent:

1. $z=x+y, x \in \mathbb{K}, y \in \mathbb{K}^{0}$, and $\langle x, y\rangle=0$;

2. $x=P_{\mathbb{K}}(z)$ and $y=P_{\mathbb{K}^{0}}(z)$.

Following Isac [26, 27], we give a new application of our main result to the so called general nonlinear complementarity problem (GNCP).

Let $S: \mathbb{K} \rightarrow 2^{\mathbb{X}}$ be a set-valued mapping. As is known [28], the GNCP with $S$ and $\mathbb{K}$, denoted by $\operatorname{GNCP}(S, \mathbb{K})$, is

$$
\operatorname{GNCP}(S, \mathbb{K}): \quad\left\{\begin{array}{l}
\text { find }(\hat{x}, \hat{y}) \in \mathbb{K} \times \mathbb{X} \\
\text { s.t. } \hat{y} \in S(\hat{x}) \cap \mathbb{K}^{\prime} \text { and }\langle\hat{x}, \hat{y}\rangle=0
\end{array}\right.
$$

Before we obtain some existence results for $\operatorname{GNCP}(S, \mathbb{K})$ by using existence results obtained in the previous sections, we give a useful theorem, which improves Theorem 4 in [26].

Theorem 5.3 The problem $\operatorname{GNCP}(S, \mathbb{K})$ has a solution if and only if the set-valued map defined, for all $x \in \mathbb{X}$, by

$$
T x=\left\{z \in \mathbb{X}, z \in P_{\mathbb{K}}(x)-S\left(P_{\mathbb{K}}(x)\right)\right\}
$$

has a fixed point in $\mathbb{X}$. Moreover, if $x_{0}$ is a fixed point of $T$, then $\hat{x}=P_{\mathbb{K}}\left(x_{0}\right)$ is a solution of the problem $\mathrm{GNCP}(S, \mathbb{K})$. 
Proof Suppose that $T$ has a fixed point $x_{0}$, that is,

$$
x_{0} \in P_{\mathbb{K}}\left(x_{0}\right)-S\left(P_{\mathbb{K}}\left(x_{0}\right)\right) .
$$

Then there exists $\hat{y} \in S\left(P_{\mathbb{K}}\left(x_{0}\right)\right)$ such that

$$
x_{0}=P_{\mathbb{K}}\left(x_{0}\right)-\hat{y} .
$$

Then if we denote by $\hat{x}=P_{\mathbb{K}}\left(x_{0}\right)$, then it is clear that $\hat{x} \in \mathbb{K}$, and by item 1 of Theorem 5.1 we get for all $x \in \mathbb{K}$,

$$
\langle\hat{y}, x\rangle=\left\langle\hat{x}-x_{0}, x\right\rangle \geq 0,
$$

then $\hat{y} \in \mathbb{K}^{\prime}$. Therefore, by item 2 of Theorem $5.1\langle\hat{y}, \hat{x}\rangle=\left\langle\hat{x}-x_{0}, \hat{x}\right\rangle=0$, which implies that $(\hat{x}, \hat{y})$ is a solution of $\operatorname{GNCP}(S, \mathbb{K})$.

Conversely, if $(\hat{x}, \hat{y})$ is a solution of $\operatorname{GNCP}(S, \mathbb{K})$, then denoting

$$
x_{0}=\hat{x}-\hat{y},
$$

by Theorem 5.2 we get $\hat{x}=P_{\mathbb{K}}\left(x_{0}\right)$, and since $\hat{y} \in S(\hat{x}) \cap \mathbb{K}^{\prime}$, we get $\hat{y} \in S\left(P_{\mathbb{K}}\left(x_{0}\right)\right)$. Hence, $x_{0} \in P_{\mathbb{K}}\left(x_{0}\right)-S\left(P_{\mathbb{K}}\left(x_{0}\right)\right)$, and thus $x_{0} \in T x_{0}$. This completes the proof.

Now we formulate an existence result for the $\operatorname{GNCP}(S, \mathbb{K})$ problem.

Theorem 5.4 Let $(\mathbb{X},\langle\cdot, \cdot\rangle)$ be a Hilbert space, and $\mathbb{K}$ be a closed convex cone in $\mathbb{X}$. Let $\left\{\varphi_{i}\right\}_{i \in I}$ be a family of lower semicontinuous functions from $\mathbb{X}$ to $\mathbb{R}_{+}$, and $a_{i}>0$ and $b_{i}>0$ be two families of positive real numbers. Suppose that the set-valued maps $T$ and $S$ defined before satisfy the supplementary condition:

For all $i \in I$ and $(x, y) \in G_{S}$, there exist $z \in T x \cap \mathbb{K}$ and $t \in S(z)$ such that

$$
\max \left\{a_{i}\|x-z\|_{\mathbb{X}}, b_{i}\|y-t\|_{\mathbb{X}}\right\} \leq \varphi_{i}(y)-\varphi_{i}(t) .
$$

Then $\operatorname{GNCP}(S, \mathbb{K})$ has a solution.

Proof It suffices to replace $T$ by $T^{\prime}$ defined from $\mathbb{K}$ into $2^{\mathbb{K}}$ as $T^{\prime}(x)=T(x) \cap \mathbb{K}$ and apply Theorem 5.3 and Proposition 3.4.

Example 5.5 Let $\mathbb{X}=\mathbb{R}, \mathbb{K}=\mathbb{R}_{+}$, and, for all $i \in I, a_{i}=b_{i}=1, \varphi_{i}(x)=|x|$ for $x \in \mathbb{X}$, and $S(x)=[0, x]$ for all $x \in \mathbb{K}$. Then the GNCP problem becomes:

$$
\operatorname{GNCP}\left(S, \mathbb{R}_{+}\right): \quad\left\{\begin{array}{l}
\text { find }(\hat{x}, \hat{y}) \in \mathbb{R}_{+} \times \mathbb{R} \\
\text { s.t. } \hat{y} \in[0, \hat{x}] \text { and } \hat{x} \hat{y}=0 .
\end{array}\right.
$$

It is obvious that $T(x)=[0, x]$ for each $(x, y) \in G_{S}$. It is clear that, for all $x \geq 0$ and $y \in[0, x]$, we get

$$
|x-y|+|y| \leq|x| \quad \Leftrightarrow \quad|x-y| \leq \varphi_{i}(x)-\varphi_{i}(y)
$$

and choosing $z \in T(x)$ and $t \in S(z)$, we have: 
1. for $x=y$, we choose $z=0$ and $t=0$, and then we have

$$
\max \{|x|,|y|\} \leq \varphi_{i}(y)
$$

2. for $y<x$, we choose $z=x-y+t$ and $t \leq \min \{x-y, y\}$, so that $|x-z|=|y-t|$, and then we get

$$
|y-t| \leq \varphi_{i}(y)-\varphi_{i}(t)
$$

Finally, by 1 and 2 we get

$$
\max \left\{a_{i}|x-z|, b_{i}|y-t|\right\} \leq \varphi_{i}(y)-\varphi_{i}(t) .
$$

Then all assumptions of Theorem 5.4 hold, and hence problem $\operatorname{GNCP}\left(S, \mathbb{R}_{+}\right)$has a solution, and the set of solutions is

$$
\operatorname{Sol}\left(\operatorname{GNCP}\left(S, \mathbb{R}_{+}\right)\right)=\{(x, 0) ; x \geq 0\} .
$$

\subsection{Differential inclusion in a nuclear space}

Let $\mathbb{R}^{d}$ (with fixed $d \in \mathbb{N}^{*}$ ), set $\mathcal{D}\left(\mathbb{R}^{d}\right)$ to be the space of all complex-valued infinitely differentiable functions on $\mathbb{R}^{d}$ with compact support, and define the differential operator for each multiindex $\alpha \in \mathbb{N}^{d}$ with $\alpha=\left(\alpha_{1}, \alpha_{2}, \ldots, \alpha_{d}\right)$ by

$$
D^{\alpha}=\frac{\partial^{|\alpha|}}{\partial x_{1}^{\alpha_{1}} \partial x_{2}^{\alpha_{2}} \cdots \partial x_{d}^{\alpha_{d}}},
$$

where $|\alpha|=\alpha_{1}+\cdots+\alpha_{d}$. The space $\mathcal{D}\left(\mathbb{R}^{d}\right)$ is endowed by a locally convex topology defined by the family of separated seminorms

$$
\|\varphi\|_{N}=\sup \left\{\left|D^{\alpha} \varphi(x)\right| ; x \in \mathbb{R}^{d} \text { and }|\alpha| \leq N\right\} .
$$

Recall that a subset $B \subset \mathcal{D}\left(\mathbb{R}^{d}\right)$ is bounded if for some compact $K \subset \mathbb{R}^{d}$, we have $B \subset$ $\mathcal{D}(K)$ and there are numbers $M_{N}<\infty$ such that every $\varphi \in B$ satisfies the inequalities

$$
\|\varphi\|_{N} \leq M_{N}, \quad N=0,1,2, \ldots
$$

It is worth noting that $\mathcal{D}\left(\mathbb{R}^{d}\right)$ endowed with the limit inductive topology of $\left\{\mathcal{D}\left(K_{n}\right)\right\}_{n}$ is a complete nonmetric space, where $\left(K_{n}\right)_{n \in \mathbb{N}}$ is an exhaustive sequence of compact subsets, that is, for every $n \in \mathbb{N}, K_{n}$ included in the interior of $K_{n+1}$, and $\mathbb{R}^{d}=\cup_{n} K_{n}$; for more details, see [29].

Now, let $\mathcal{D}^{\prime}\left(\mathbb{R}^{d}\right)$ be the strong dual of $\mathcal{D}\left(\mathbb{R}^{d}\right)$, also endowed with the locally convex topology generated by an uncountable separated family of seminorms over the bounded subset of $\mathcal{D}\left(\mathbb{R}^{d}\right)$ denoted by $\tau$, that is,

$$
p_{B}(f)=\sup _{\varphi \in B}|\langle f, \varphi\rangle|, \quad B \subset \mathcal{D}\left(\mathbb{R}^{d}\right) \quad \text { bounded. }
$$


Definition 5.6 In a Hausdorff locally convex space $\left(\mathbb{X},\left\{p_{i}\right\}_{i \in \Lambda}\right)$, a convex cone $\mathbb{K} \subset \mathbb{X}$ is supernormal [13] if for each $i \in \Lambda$, there exists a continuous linear form $f_{i} \in \mathbb{K}^{\prime}$ (dual cone) such that, for each $x \in \mathbb{K}$, we have

$$
p_{i}(x) \leq f_{i}(x)
$$

$\mathcal{D}^{\prime}\left(\mathbb{R}^{d}\right)$ endowed with $\tau$-topology is a nuclear space [17], and we have the following:

Proposition 5.7 In a nuclear space $\mathbb{X}$, a convex cone $\mathbb{K} \subset \mathbb{X}$ is $\tau$-supernormal if and only if it is $\tau$-normal.

It is shown in [17] that the cone $\mathbb{K}$ defined by

$$
\mathbb{K}=\left\{\Lambda \in \mathcal{D}^{\prime}\left(\mathbb{R}^{d}\right) ;\langle\Lambda, \varphi\rangle \geq 0, \forall \varphi \in \mathcal{C}\right\}
$$

is $\tau$-normal cone, where $\mathcal{C}=\left\{\varphi \in \mathcal{D}\left(\mathbb{R}^{d}\right) ; \varphi(x) \geq 0, \forall x \in \mathbb{R}^{d}\right\}$, and hence $\mathbb{K}$ is $\tau$-supernormal.

Next, we propose to solve the partial differential inclusion problem;

$$
(\mathcal{P}):\left\{\begin{array}{l}
\text { find a locally integrable function } u \in L_{\text {loc }}^{1}\left(\mathbb{R}^{d}\right) \text { such that } \\
D^{\alpha} u \in F(u) \text { a.e. on } \mathbb{R}^{d}
\end{array}\right.
$$

where $\alpha \in \mathbb{N}^{d}$ a multiindex, and $\left.F: L_{\text {loc }}^{1}\left(\mathbb{R}^{d}\right) \longrightarrow 2^{L_{\mathrm{loc}}^{1}} \mathbb{R}^{d}\right)$.

Given $u \in L_{\text {loc }}^{1}\left(\mathbb{R}^{d}\right)$, it is shown in [29] that $u$ defines a regular distribution, denoted $\Lambda_{u} \in \mathcal{D}^{\prime}\left(\mathbb{R}^{d}\right)$, as follows:

$$
\Lambda_{u}(\varphi)=\int_{\mathbb{R}^{d}} u(x) \varphi(x) d x
$$

for all $\varphi \in \mathcal{D}\left(\mathbb{R}^{d}\right)$.

Also, if $u \in L_{\text {loc }}^{1}\left(\mathbb{R}^{d}\right)$, we know that $\Lambda_{D^{\alpha} u}=D^{\alpha} \Lambda_{u}$, and hence we propose to solve problem $(\mathcal{P})$ in regular distributions setting and consider the differentiability in the weak sense. Problem $(\mathcal{P})$ is transformed by the canonical isomorphism

$$
\mathcal{G}: L_{\mathrm{loc}}^{1}\left(\mathbb{R}^{d}\right) \longrightarrow \mathcal{G}\left(\mathcal{D}^{\prime}\left(\mathbb{R}^{d}\right)\right)
$$

to

$$
\left(\mathcal{P}^{\prime}\right):\left\{\begin{array}{l}
\text { find a regular distribution } \Lambda_{u} \in \mathcal{D}^{\prime}\left(\mathbb{R}^{d}\right) \text { such that } \\
D^{\alpha} \Lambda_{u} \in \mathcal{F}\left(\Lambda_{u}\right) \text { a.e. on } \mathbb{R}^{d},
\end{array}\right.
$$

where $\mathcal{F}$ is the set-valued map defined from $\mathcal{D}^{\prime}\left(\mathbb{R}^{d}\right)$ into $2^{\mathcal{D}^{\prime}\left(\mathbb{R}^{d}\right)}$ by

$$
\Lambda_{v} \in \mathcal{F}\left(\Lambda_{u}\right) \quad \Leftrightarrow \quad v \in F(u) .
$$

Now, passing to the second part of our developments, there is no chance that problem $\left(\mathcal{P}^{\prime}\right)$ has a solution, so we will give a sufficient condition on the set-valued map $F$ in order 
that the problem has at least one solution. For this, we define two subsets $\mathcal{I}$ and $\mathcal{J}$ of $\mathcal{D}^{\prime}\left(\mathbb{R}^{d}\right)$ by

$$
\begin{aligned}
& \mathcal{I}=\left\{\Lambda_{f} ; f \in L_{\mathrm{loc}}^{1}\left(\mathbb{R}^{d}\right), \Lambda_{f}(\varphi)=\int_{\mathbb{R}^{d}} f(x) D^{\alpha} \varphi(x) d x \text { for each } \varphi \in \mathcal{D}\left(\mathbb{R}^{d}\right)\right\} ; \\
& \forall \Lambda_{u} \in \mathcal{D}^{\prime}\left(\mathbb{R}^{d}\right): \quad \mathcal{J}\left(\Lambda_{u}\right)=\left\{\Lambda_{f} \in \mathcal{I} ; u(x) \geq(-1)^{|\alpha|} f(x), \forall x \in \mathbb{R}^{d}\right\}
\end{aligned}
$$

and for each regular distribution $\Lambda_{u} \in \mathcal{D}^{\prime}\left(\mathbb{R}^{d}\right)$, we define the set-valued maps $\mathcal{R}$ and $\mathcal{T}$ as follows:

$$
\begin{aligned}
& \mathcal{R}\left(\Lambda_{u}\right)=\left\{\Lambda_{v} \in \mathcal{D}^{\prime}\left(\mathbb{R}^{d}\right) ; \forall \varphi \in \mathcal{C},\left\langle\Lambda_{u}-\Lambda_{v}, \varphi\right\rangle \geq 0\right\} \\
& \mathcal{T}\left(\Lambda_{u}\right)=\left\{\Lambda_{v} \in \mathcal{R}\left(\Lambda_{u}\right) ; D^{\alpha} v \in F(u) \text { a.e. on } \mathbb{R}^{d}\right\}
\end{aligned}
$$

It is obvious that $\mathcal{R}\left(\Lambda_{u}\right)$ is nonempty since $\Lambda_{u} \in \mathcal{R}\left(\Lambda_{u}\right)$, and for $\mathcal{T}\left(\Lambda_{u}\right)$, we need the next lemma.

Lemma 5.8 If for each $\Lambda_{u} \in \mathcal{D}^{\prime}\left(\mathbb{R}^{d}\right), \mathcal{F}\left(\Lambda_{u}\right) \cap \mathcal{J}\left(\Lambda_{u}\right) \neq \emptyset$, then $\mathcal{T}\left(\Lambda_{u}\right)$ is a nonempty subset of $\mathcal{D}^{\prime}\left(\mathbb{R}^{d}\right)$.

Proof Let $f$ be a locally integrable function, and let $\Lambda_{u} \in \mathcal{D}^{\prime}\left(\mathbb{R}^{d}\right)$. Then the function

$$
\varphi \mapsto \int_{\mathbb{R}^{d}} f(x) D^{\alpha} \varphi(x) d x \quad \text { is an element of } \mathcal{F}\left(\Lambda_{u}\right),
$$

and a simple calculation leads to

$$
\begin{aligned}
\int_{\mathbb{R}^{d}} f(x) D^{\alpha} \varphi(x) d x & =(-1)^{|\alpha|} \int_{\mathbb{R}^{d}} D^{\alpha} f(x) \varphi(x) d x \\
& =\int_{\mathbb{R}^{d}} D^{\alpha}\left[(-1)^{|\alpha|} f(x)\right] \varphi(x) d x .
\end{aligned}
$$

Put $v(x)=(-1)^{|\alpha|} f(x)$ for $x \in \mathbb{R}^{d}$. Then $v \in L_{\text {loc }}^{1}\left(\mathbb{R}^{d}\right)$, and

$$
\int_{\mathbb{R}^{d}} D^{\alpha} v(x) \varphi(x) d x=\Lambda_{D^{\alpha} v}(\varphi)
$$

which leads to $\Lambda_{D^{\alpha}{ }_{v}} \in \mathcal{F}\left(\Lambda_{u}\right)$. Thus, $D^{\alpha} v \in F(u)$.

For each $\varphi \in \mathcal{C}$, we have

$$
\begin{aligned}
\Lambda_{u}(\varphi)-\Lambda_{v}(\varphi) & =\Lambda_{u}(\varphi)-(-1)^{|\alpha|} \Lambda_{f}(\varphi) \\
& =\int_{\mathbb{R}^{d}}\left[u(x)-(-1)^{|\alpha|} f(x)\right] \geq 0 .
\end{aligned}
$$

Hence, $\Lambda_{v} \in \mathcal{T}\left(\Lambda_{u}\right)$.

As an interesting application of the main result, we can state and prove the following existence theorem. 
Theorem 5.9 If $\mathbb{K}$ and $\mathcal{R}$ are as before and $\mathcal{T}$ satisfies the assumption in the previous lemma, then problem $\left(\mathcal{P}^{\prime}\right)$ has a solution.

Proof By assumption, for each $\Lambda_{u} \in \mathcal{D}^{\prime}\left(\mathbb{R}^{d}\right)$, there exists $\Lambda_{v} \in \mathcal{T}\left(\Lambda_{u}\right)$ such that:

(i) $D^{\alpha} \Lambda_{v} \in \mathcal{F}\left(\Lambda_{u}\right)$, and

(ii) $\Lambda_{v} \in \mathcal{R}\left(\Lambda_{u}\right)$.

Then, for every $\varphi \in \mathcal{D}\left(\mathbb{R}^{d}\right)$, we have

$$
\left\langle\Lambda_{u}-\Lambda_{v}, \varphi\right\rangle \geq 0 \quad \Longleftrightarrow\left(\Lambda_{u}-\Lambda_{v}\right)(\varphi) \geq 0,
$$

which implies that $\left(\Lambda_{u}-\Lambda_{v}\right) \in \mathbb{K}$; since $\mathbb{K}$ is a supernormal cone, for each bounded subset $B$ of $\mathcal{D}\left(\mathbb{R}^{d}\right)$, there exists $f_{B} \in \mathbb{K}^{\prime}$ such that

$$
p_{B}\left(\Lambda_{u}-\Lambda_{v}\right) \leq f_{B}\left(\Lambda_{u}-\Lambda_{v}\right) \quad \Longleftrightarrow \quad p_{B}\left(\Lambda_{u}-\Lambda_{v}\right) \leq f_{B}\left(\Lambda_{u}\right)-f_{B}\left(\Lambda_{v}\right) .
$$

All assumptions of our former result in Proposition 3.4 hold. Therefore, $\mathcal{T}$ has a fixed point $\Lambda_{u^{\star}} \in \mathcal{D}^{\prime}\left(\mathbb{R}^{d}\right)$, that is,

$$
\Lambda_{u^{\star}} \in \mathcal{T}\left(\Lambda_{u^{\star}}\right) \quad \Leftrightarrow \quad D^{\alpha} u^{\star} \in F\left(u^{\star}\right) .
$$

\section{Competing interests}

The authors declare that they have no competing interests.

\section{Authors' contributions}

All the authors contributed equally. All authors read and approved the final manuscript.

\section{Acknowledgements}

The authors are grateful to the anonymous referees for their helpful comments and remarks.

Received: 6 August 2016 Accepted: 13 January 2017 Published online: 01 February 2017

\section{References}

1. Ekeland, I: On the variational principle. J. Math. Anal. Appl. 47, 324-353 (1974)

2. Cammaroto, F, Chinni, A, Sturiale, G: A remark on Ekeland's principle in locally convex topological vector spaces. Math. Comput. Model. 30(9), 75-79 (1999)

3. Isac, G: Ekeland's principle and nuclear cones: a geometrical aspect. Math. Comput. Model. 26(11), 111-116 (1997)

4. Chen, GY, Huang, XX, Hou, SH: General Ekeland's variational principle for set-valued mappings. J. Optim. Theory Appl, 106(1), 151-164 (2000)

5. Isac, G, Tammer, C: Nuclear and full nuclear cones in product spaces: Pareto efficiency and an Ekeland type variational principle. Positivity 9(3), 511-539 (2005)

6. Cammaroto, F, Chinni, A, Sturiale, G: On an extension of Ekeland's principle for vector-valued functions. Optimization 43(1), 19-28 (1998)

7. Göpfert, A, Tammer, C, Zălinescu, C: On the vectorial Ekeland's variational principle and minimal points in product spaces. Nonlinear Anal., Theory Methods Appl. 39(7), 909-922 (2000)

8. Brezis, H, Browder, FE: A general principle on ordered sets in nonlinear functional analysis. Adv. Math. 21, 355-364 (1976)

9. Zermelo, EB: Dass jede Menge wohlgeordnet werden kann. Math. Ann. 59(4), 514-516 (1904)

10. Hamel, A, Löhne, A: Minimal point theorem in uniform spaces. Univ., Fachbereich Mathematik und Informatik (2002)

11. Fang, J-X: The variational principle and fixed point theorems in certain topological spaces. J. Math. Anal. Appl. 202(2), 398-412 (1996)

12. Isac, G: Un théorème de point fixe de type Caristi dans les espaces localement convexes. Applications. Zbornik Radova, Review of Research (1985)

13. Isac, G: Supernormal cones and fixed point theory. J. Math. 17(3) (1987)

14. Caristi, J: Fixed point theorems for mappings satisfying inwardness conditions. Trans. Am. Math. Soc. 215, 241-251 (1976)

15. Downing, D, Kirk, AW: Generalization of Caristi's theorem with applications to nonlinear mapping theory. Pac. J. Math. 69(2), 339-346 (1977)

16. Włodarczyk, K, Plebaniak, R, Doliński, M: Cone uniform, cone locally convex and cone metric spaces, endpoints, set-valued dynamic systems and quasi-asymptotic contractions. Nonlinear Anal., Theory Methods Appl. 71(10), 5022-5031 (2009) 
17. Schaefer, HH: Topological Vector Spaces. Springer, New York (1971)

18. Peressini, AL: Ordered Topological Vector Spaces. Harper \& Row, New York (1967)

19. Chen, $G Y$, Yang, $X Q, Y u, H$ : A nonlinear scalarization function and generalized quasi-vector equilibrium problems. J. Glob. Optim. 32(4), 451-466 (2005)

20. Chen, GY, Huang, XX, Yang, XQ: Vector Optimization: Set-Valued and Variational Analysis. Springer, Berlin (2006)

21. Du, W-S: On some nonlinear problems induced by an abstract maximal element principle. J. Math. Anal. Appl. 347, 391-399 (2008)

22. Tammer, C, Weidner, P: Nonconvex separation theorems and some applications in vector optimization. J. Optim. Theory Appl. 67, 297-320 (1990)

23. Göpfert, A, Tammer, C, Riahi, H, Zălinescu, C: Variational Methods in Partially Ordered Spaces. Springer, New York (2003)

24. Du, W-S: A note on cone metric fixed point theory and its equivalence. Nonlinear Anal., Theory Methods Appl. 72(5), 2259-2261 (2010)

25. Kadelburg, Z, Radenović, S, Rakočević, V: A note on the equivalence of some metric and cone metric fixed point results. Appl. Math. Lett. 24(3), 370-374 (2011)

26. Isac, G: Equivalence between nonlinear complementarity problem and fixed point problem. In: Encyclopedia of Optimization, 2nd edn., pp. 563-567 (2001)

27. Isac, G, Németh, AB: Projection methods, isotone projection cones, and the complementarity problem. J. Math. Anal. Appl. 153(1), 258-275 (1990)

28. Cubiotti, P, Yao, J-C: Multivalued (S) ${ }_{+}^{1}$ operators and generalized variational inequalities. Comput. Math. Appl. 29(12), 49-56 (1995)

29. Rudin, W: Functional Analysis. Internat. Ser. Pure Appl. Math. McGraw-Hill, New York (1991)

\section{Submit your manuscript to a SpringerOpen ${ }^{\odot}$ journal and benefit from:}

- Convenient online submission

Rigorous peer review

- Immediate publication on acceptance

- Open access: articles freely available online

- High visibility within the field

- Retaining the copyright to your article 